\title{
The impact of COVID-19 control measures on social contacts and transmission in Kenyan informal settlements
}

\author{
Matthew Quaife ${ }^{1,2^{*}}$ D, Kevin van Zandvoort', Amy Gimma', Kashvi Shah², Nicky McCreesh', Kiesha Prem',

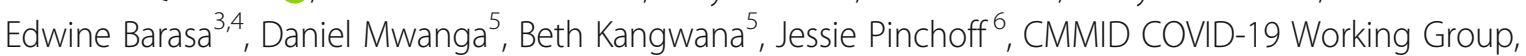 \\ W. John Edmunds ${ }^{1}$, Christopher I. Jarvis ${ }^{1}$ and Karen Austrian ${ }^{5}$
}

\begin{abstract}
Background: Many low- and middle-income countries have implemented control measures against coronavirus disease 2019 (COVID-19). However, it is not clear to what extent these measures explain the low numbers of recorded COVID-19 cases and deaths in Africa. One of the main aims of control measures is to reduce respiratory pathogen transmission through direct contact with others. In this study, we collect contact data from residents of informal settlements around Nairobi, Kenya, to assess if control measures have changed contact patterns, and estimate the impact of changes on the basic reproduction number $\left(R_{0}\right)$.

Methods: We conducted a social contact survey with 213 residents of five informal settlements around Nairobi in early May 2020, 4 weeks after the Kenyan government introduced enhanced physical distancing measures and a curfew between $7 \mathrm{pm}$ and $5 \mathrm{am}$. Respondents were asked to report all direct physical and non-physical contacts made the previous day, alongside a questionnaire asking about the social and economic impact of COVID-19 and control measures. We examined contact patterns by demographic factors, including socioeconomic status. We described the impact of COVID-19 and control measures on income and food security. We compared contact patterns during control measures to patterns from non-pandemic periods to estimate the change in $R_{0}$.
\end{abstract}

Results: We estimate that control measures reduced physical contacts by $62 \%$ and non-physical contacts by either $63 \%$ or $67 \%$, depending on the pre-COVID-19 comparison matrix used. Masks were worn by at least one person in $92 \%$ of contacts. Respondents in the poorest socioeconomic quintile reported 1.5 times more contacts than those in the richest. Eighty-six percent of respondents reported a total or partial loss of income due to COVID-19, and $74 \%$ reported eating less or skipping meals due to having too little money for food.

(Continued on next page)

\footnotetext{
* Correspondence: matthew.quaife@lshtm.ac.uk

'Faculty of Epidemiology and Population Health, London School of Hygiene and Tropical Medicine, London, UK

${ }^{2}$ Faculty of Public Health and Policy, London School of Hygiene and Tropical Medicine, London, UK

Full list of author information is available at the end of the article
}

(c) The Author(s). 2020 Open Access This article is licensed under a Creative Commons Attribution 4.0 International License, which permits use, sharing, adaptation, distribution and reproduction in any medium or format, as long as you give appropriate credit to the original author(s) and the source, provide a link to the Creative Commons licence, and indicate if changes were made. The images or other third party material in this article are included in the article's Creative Commons licence, unless indicated otherwise in a credit line to the material. If material is not included in the article's Creative Commons licence and your intended use is not permitted by statutory regulation or exceeds the permitted use, you will need to obtain permission directly from the copyright holder. To view a copy of this licence, visit http://creativecommons.org/licenses/by/4.0/. The Creative Commons Public Domain Dedication waiver (http://creativecommons.org/publicdomain/zero/1.0/) applies to the data made available in this article, unless otherwise stated in a credit line to the data. 
(Continued from previous page)

Conclusion: COVID-19 control measures have had a large impact on direct contacts and therefore transmission, but have also caused considerable economic and food insecurity. Reductions in $R_{0}$ are consistent with the comparatively low epidemic growth in Kenya and other sub-Saharan African countries that implemented similar, early control measures. However, negative and inequitable impacts on economic and food security may mean control measures are not sustainable in the longer term.

Keywords: COVID-19, SARS-CoV-2, Social contacts, Physical distancing

\section{Background}

Over 18.3 million cases and 694,000 deaths from COVID-19 have been recorded worldwide as of 4 August 2020 [1]. Most recorded cases and deaths have occurred in high-income countries in Europe and North America. Many countries introduced extreme physical distancing control measures to control SARS-CoV-2 transmission [2]. Modelling studies suggest that without substantial mitigation measures, most low- and middle-income (LMIC) settings, including sub-Saharan Africa, will experience a delayed, but severe epidemic $[3,4]$. Yet to-date, the numbers of recorded cases and deaths in Africa are much lower than predictions, prompting speculation on why many African countries have so far avoided a severe uncontrolled epidemic. A range of reasons has been proposed, including differences between settings in case and death detection capacity, demographic factors such as population age distribution, and the role of temperature and aridity in transmission [5-10]. However, many sub-Saharan African countries implemented lockdown and curfew measures far earlier in their country's epidemic trajectories than most higher-income settings in Europe and North America. For example, Kenya-the focus of the current studyimplemented a partial lockdown on 6 April 2020 when the country had recorded just 158 cases and 6 deaths. In contrast, although case detection rates may differ between settings, the UK implemented its own lockdown on 23 March 2020 after recording 6650 cases and 335 deaths $[1,2]$. The first reported case in Kenya was on 13 March 2020, and schools closed on 15 March 2020. Suspension of international flights, including mandatory quarantine of incoming residents; closure of bars and restrictions on restaurant opening hours; and a ban on large gatherings were imposed on 25 March 2020, soon followed by an enactment of a nationwide curfew from $7 \mathrm{pm}$ to $5 \mathrm{am}$. On 5 April 2020, the Kenyan government declared wearing face masks as mandatory in any public place. Recently, cessation of movement was imposed in informal settlements in Mombasa and Nairobi, following a rise in cases in Nairobi's Kibera informal settlement. Consequently, the government has indicated additional physical distancing measures may be authorised.

Physical distancing control measures seek to reduce the number of contacts between people where transmission could occur. To predict the impact of control measures accurately, quantitative data on the number and type of contacts between people is required. Todate, only a few empirical studies have been published to assess the impact of COVID-19 control measures on contacts; these have been conducted in China [11], the USA [12], and Europe [13]; but none were undertaken in sub-Saharan Africa. In fact, prior to the current pandemic, a systematic review [14] reported that just four social contact surveys out of 64 had been conducted in sub-Saharan Africa, including one in Kenya [15-17]. To our knowledge, just one LMIC study has been published since this review [18]. This lack of evidence means that many SARSCoV-2 transmission models primarily use synthetic contact matrices for LMIC settings, which use demographic, household composition, classroom size, and other data to adjust social contact data from primarily high-income settings $[19,20]$. Although one social mixing study was conducted in Kilifi, a coastal area of Kenya [21], outside of one study which collected data from a South African township [16], no published contact data exist from informal settlements, which may be particularly vulnerable to COVID-19 due to high levels of population density, indoor crowding, and household sizes, alongside intergenerational mixing within the household.

Between-person contacts drive the transmission of respiratory pathogens, such as SARS-CoV-2. Understanding how contact patterns change under different control measures is important to inform decisions on whether and how to implement them. In this study, we describe a survey of contact patterns conducted among a sample of adults from five informal settlements in urban and peri-urban areas around Nairobi. We explore how direct contacts vary across respondent characteristics, including by socioeconomic status. We estimate the impact of current control measures on the reproduction number, $R_{0}$, to evaluate whether these measures might be sufficient to control the epidemic. We also describe income 
losses and food security that respondents attribute to COVID-19 and control measures.

\section{Methods \\ Ethics}

Participation in the study was voluntary, and analyses were conducted on anonymised data. The study was approved by the internal review board of the Population Council (study number 936), the ethics committee of the London School of Hygiene and Tropical Medicine (reference number 22294), and the AMREF Health Africa Ethics and Scientific Review Committee in Kenya (P803/ 2020).

\section{Survey methodology}

Adult respondents were recruited from two existing Population Council cohorts in five informal settlements around Nairobi (Kibera, Huruma, Kariobangi, Dandora, and Mathare). The existing cohorts were part of the Adolescent Girls Initiative Kenya (AGI-K) and Nisikilize Tujengane (NISITU - Listen to Me, Let us Grow Together) studies. The cohorts were in place to study the impacts of multi-sectoral interventions on adolescents, and consisted of randomly selected households from informal settlements which contained at least one adolescent in January 2015 (AGI-K) or January 2018 (NISITU). In May 2020, 1750 respondents from AGI-K and NISI TU cohorts completed a telephone survey on COVID-19 knowledge, attitudes, and perceptions (KAP). Of these 1750, an age- and sex-stratified random sample of 213 respondents completed a contact survey. Stratification was based on 2019 Kenya census data for Nairobi county, with a target sample size of 200 and $20 \%$ oversampling to account for refusal. This was based on the sample sizes of similar contact surveys [14], alongside feasibility of phone interviewing during lockdown. Background data, including household ownership of assets, were merged from previous survey rounds. Respondents were first asked a range of questions on COVID-19 including knowledge and experience of testing and symptoms, economic impacts on the household, and food availability and cost. Then, respondents were asked to report all direct physical and non-physical contacts made between $5 \mathrm{am}$ the day preceding the survey and 5 am the day of the survey. A direct contact was defined as someone respondents met in person and with whom they had either (i) "physical contact (any sort of skin-toskin contact e.g. a handshake, embracing, kissing, sleeping on the same bed/mat/blanket, sharing a meal together out of the same bowl, playing football or other contact sports, sitting next to someone while touching shoulder to shoulder, etc.", or (ii) "Non-physical contact (you did not touch the person, but exchanged at least a few words, face-to-face within 2 metres - for example, someone you bought something from in the market, or rode with on a minibus, or worked with in the same area)". All respondents were over the age of 18 , so no contact data were collected from children; however, respondents were able to list contacts under the age of 18 .

We made pragmatic adaptations to existing contact measurement tools to allow them to be conducted over the phone, primarily to reduce respondent burden and to ensure that aggregate contact data were not biassed downwards by respondent fatigue. Respondents were first asked about contacts with members of their household the previous day, recording the contact age, gender, and whether contacts were physical or non-physical. Then, respondents were asked how many nonhousehold contacts they had had in the same timeframe. Those who reported nine or fewer outside-household contacts were asked to describe each contact's age, gender, whether the contact was physical or non-physical, the duration of the contact, and whether a mask was worn by the respondent or contact. Those who reported ten or more outside-household contacts were asked how many of these contacts were physical/non-physical, in the age ranges under $18,18-60$, and over 60 . The contact tool is shown in Additional file 1.

\section{Statistical analysis}

$R$ version 4.0.0 and Stata 15 were used for analyses; the code and data are publicly available at https://github. com/mquaife/kenya_mixing. The age and gender of respondents were compared to the full sample from which they were drawn, alongside census data to assess the representativeness of the sample. Data on household assets were used to classify respondents into wealth quintiles using principal component analysis; Additional file 2 gives information on this, alongside methods used to estimate economic and food security.

We calculated the mean number of social contacts per person per day, stratified by respondent age, sex, household size, and education level. We then calculated social contact matrices for the age category-specific daily frequency of direct contacts, adjusting for contact reciprocity and the age distribution using census data from informal settlement sub-counties. We then compared the mean total number of daily contacts by age group to the only empirical dataset available from Kenya in Kiti et al. [21], alongside synthetic matrices from 2017 [19] and 2020 [20]. Kiti et al. collected data on physical contacts only, so we restrict our sample to physical contacts when comparing with this study. We adjusted both matrices to match the age structure of the informal settlement setting, using the 2019 Kenyan Population and Housing Census to adjust from Kilifi and nationally representative populations, respectively [22]. Additional file 3 provides more detail. Because Kiti et al. 
collected data on the age of contacts in categories $(<1$, $1-5,6-15,16-19,20-49,50+)$ which were different to those in this survey, we restructured both age matrices and used 1000 bootstrapped samples of both datasets to impute the number of contacts for matching age ranges. We adjusted for symmetry after bootstrapping because one age range in our data $(60+)$ had fewer than five respondents. Bootstrapping was not possible with Prem et al. matrices as they do not relate to individual level data.

As respondents under the age of 18 were not included as survey respondents, we imputed child contacts using methods developed by Klepac et al. [23], and implemented for the same purpose in a UK study [13]. This involved taking the ratio of the dominant eigenvalues between our matrices and the comparable setting-adjusted matrices to scale missing matrix elements.

Finally, we estimated the impact of control measures on the basic reproduction number $\left(R_{0}\right)$ in this population. Because there are no baseline contact data from this population without control measures, we assume that contact patterns in this sample prior to control measures were similar to those estimated by Kiti et al. or Prem et al. We make the common assumption for respiratory infections that the next-generation matrix is a function of the age-specific number of contacts, the percontact transmission probability, and the duration of infectiousness, and that $R_{0}$ is therefore proportional to the dominant eigenvalue of the contact matrix $[17,18]$. We assume that existing matrices are comparable to the informal settlement setting of this study after adjusting for age distribution, that there were no changes in the duration of infectiousness during the study period, that percontact transmission probability also remained constant, and that all age groups have the same per-contact transmission probability, given infection. With these assumptions, the relative reduction in $R_{0}$ can be estimated as the reduction in the dominant eigenvalue of the contact matrices. Our central estimate of the $R_{0}$ of SARS-CoV-2 is $2.6(\mathrm{SD}=0.54)$, as estimated in a meta-analysis of published estimates of $R_{0}$ prior to the introduction of control measures [13]. Because studies in this meta-analysis were predominantly based on European and Asian countries, we explore a lower bound of $1.46(\mathrm{SD}=0.38)$ based on the earliest estimate of the time-varying reproduction number in Kenya [24]. We also use a higher bound $R_{0}$ of $3.8(\mathrm{SD}=0.71)$ based on modelling analyses from European countries [25]. Finally, although there is limited evidence of age-specific variation in infectiousness or symptomatic rate given infection, there is some evidence that children are around half as susceptible to SARSCoV-2 infection compared to adults [26]. In a sensitivity analysis, we explore whether this impacts $R_{0}$ estimates.

\section{Results}

\section{Respondent and contact characteristics}

Out of the 1970 people sampled for the KAP survey, 1745 interviews were completed. Of the initial 1970 sampled, 237 were sampled to complete the additional contacts module. In total, 213 were successfully interviewed and recorded 3809 contacts. Eight hundred thirty (22\%) of these were household contacts, and 324 (9\%) were non-household contacts on which we have detailed information. The remaining 2655 (70\%) were nonhousehold contacts of respondents who reported ten or more such contacts. The mean age of respondents was 33 (SD 11.38, max 70), and 51\% were female (108/213). Table 1 shows that the age and gender distribution of respondents broadly matched that of (a) the sample from which respondents were randomly chosen and (b) the Kenyan adult population. Compared to both groups, there is some indication that our sample has more 1829 year olds and fewer $60+$ year olds than national data, whilst our sample is substantially older than that of Kiti et al.

\section{Implications of COVID-19 control measures}

Eight respondents (4\%) reported two or more COVID19 symptoms $^{1}$ in the previous 7 days. Forty-two percent of respondents $(89 / 213)$ thought they had a high chance of acquiring SARS-CoV-2, and 81\% (172/213) thought the implications would be "severe" or "very severe" if they caught the virus. When asked an open-ended question without prompting what they would do if they developed COVID-19 symptoms, 64\% (136/213) thought they would take a test, and 7\% (16/213) said they would stay at home or avoid social gatherings. Just $6 \%(13 / 213)$ of respondents knew someone either who was suspected of having COVID-19 or who had tested positive.

Respondents reported substantial food and economic insecurity due to COVID-19 and control measures. Around a third $(36 \%, 76 / 213)$ reported the pandemic had caused a complete loss of income, and an additional $50 \%(107 / 213)$ reported partial income losses. Eightythree percent (177) reported experiencing increases in food prices, and three quarters of respondents reported eating less or skipping meals due to having too little money for food $(74 \%, 158 / 213)$; all but one $(157 / 158)$ reported that this was due to the situation with COVID19. Just $21 \%(44 / 213)$ reported receiving monetary or non-monetary assistance in the previous 7 days $-78 \%$ (166) reported that food was the one of the biggest needs that was currently unmet.

${ }^{1}$ Fever, headache, cough, diarrhoea, difficulty breathing, loss of taste or smell, tiredness/fatigue, chest pain, chills, rash, dizziness, sneezing, sore throat, myalgia 
Table 1 Respondent characteristics in this study and comparison with data from mixing module respondents, full sample, and Kenya national demographics

\begin{tabular}{|c|c|c|c|c|c|c|c|}
\hline \multirow{2}{*}{$\overline{\text { Age group }}$} & \multicolumn{2}{|c|}{$\begin{array}{l}\text { Respondents in this survey } \\
(n=213)\end{array}$} & \multirow[t]{2}{*}{ KAP survey respondents $(n=1745)$} & \multirow[t]{2}{*}{ Kenya* (all ages) } & \multirow[t]{2}{*}{ Kenya* (> 19 only) } & \multicolumn{2}{|c|}{ Kiti et al. [21] } \\
\hline & & & & & & Age grou & \\
\hline $0-17$ & 0 & - & - & $50 \% *$ & & $<1$ & $15 \%$ \\
\hline $18-29$ & 95 & $44 \%$ & $28 \%$ & $18 \% *$ & $36 \% *$ & $1-5$ & $16 \%$ \\
\hline $30-39$ & 61 & $29 \%$ & $30 \%$ & $14 \%$ & $28 \%$ & $6-14$ & $17 \%$ \\
\hline $40-49$ & 34 & $16 \%$ & $29 \%$ & $9 \%$ & $18 \%$ & $15-19$ & $16 \%$ \\
\hline $50-59$ & 21 & $10 \%$ & $10 \%$ & $5 \%$ & $10 \%$ & $20-49$ & $24 \%$ \\
\hline $60+$ & 3 & $1 \%$ & $3 \%$ & $4 \%$ & $8 \%$ & $50+$ & $11 \%$ \\
\hline \multicolumn{8}{|l|}{ Gender } \\
\hline Male & 106 & $50 \%$ & $37 \%$ & $50 \%$ & $51 \%$ & Male & $46 \%$ \\
\hline Female & 108 & $50 \%$ & $63 \%$ & $50 \%$ & $49 \%$ & Female & $54 \%$ \\
\hline
\end{tabular}

*Kenyan national data from United Nations World Population Prospects [19]

COVID-19 control measures meant 92\% (196/213) of respondents reported seeing friends less, and 64\% (136) seeing family less. Twenty-five percent of respondents $(54 / 213)$ reported leaving the settlement where the interview was conducted in the previous $24 \mathrm{~h}$. At the time of data collection, mask wearing was required by the Kenyan government in public places and was very common: 94\% (199/211) of respondents reported "always" wearing a mask outside of their house.

\section{Contact patterns}

The mean number of contacts reported was 18 (median 13, IQR 7-23), 4 household contacts (median 4, IQR 312 ) and 15 non-household contacts (median 10, IQR 420). As shown in Fig. 1, respondents in the poorest quintile reported 1.5 times as many contacts as those in the richest quintile and we find evidence of a downwards trend in contacts as socioeconomic status increases (non-parametric test for trend $p=0.02$ ). There was weak evidence that men had more contacts than women $(20.3$ $-15.5=4.8, t$ test $p=0.04)$ and contacts increased with age (non-parametric test for trend $p=0.05$ ). Just $22 \%$ $(847 / 3841)$ of contacts were reported within the household, and total contacts did not vary substantially by household size or by respondent education level. This lack of variation by household size is consistent with most contacts being outside of the household.

Figure 2 summarises the characteristics of contacts for which we have detailed information (830 household contacts and 324 non-household contacts where a respondent reported fewer than ten non-household contacts). Most physical contacts were household contacts, and the proportion of female contacts was higher among household than non-household contacts. Just $8 \%(27 / 324)$ of non-household contacts took place without a mask being worn by either the respondent or the contact. Most reported non- household contacts were brief: 40\% (130/324) were under $5 \mathrm{~min}$, and a further $23 \%(75 / 324)$ between 5 and $15 \mathrm{~min}$. Finally, 41\% (133/324) of non-household contacts took place in an outside location, and 34\% $(110 / 324)$ of non-household contacts were in the home of the respondent or contact. Figure 3 shows age-specific contact matrices disaggregated by contact location and type; these are asymmetric and not adjusted for demography. Matrices are consistent with the majority of contacts occurring outside of the household and being non-physical.

Figure 4 uses the two existing contact matrices for Kenya to impute contact patterns for under $18 \mathrm{~s}$, adjusting for age-distribution and symmetry. The two pre-COVID-19 data sources differ substantially in their methods, and the differences are propagated in these adjusted matrices. We find a $62 \%$ reduction in physical contacts, and a $63-67 \%$ reduction in all contacts compared to before the epidemic. We estimate $R_{0}$ under control measures, shown in Fig. 5. All comparisons to pre-COVID-19 matrices assuming $R_{0}=2.64$ suggest that control measures reduced $R_{0}$ to below one, to 0.6 (IQR $0.50,0.68$ ) for physical contacts and to either 0.54 (IQR $0.46,0.61$ ) or 0.67 (IQR $0.57,0.76$ ) depending the synthetic matrix used as comparator, based on Prem et al. 2017 [19] and 2020 [20], respectively. Using the lower $R_{0}$ estimate of 1.46 , we estimate reductions to 0.33 (IQR 0.27 , 0.39 ) for physical contacts, and either 0.30 (IQR $0.24,0.35$ ) or 0.37 (IQR $0.3,0.43$ ) all contacts. Using the higher $R_{0}$ of 3.8 , we estimate reductions to 0.88 (IQR 0.76, 0.99) for physical contacts, and either 0.79 (IQR $0.69,0.89$ ) or 0.98 (IQR $0.86,1.1$ ) all contacts. Based on these values, control measures would have reduced the mean estimate of $R_{0}$ to below one even if the initial $R_{0}$ had been as high as 4.36 assuming only physical contacts lead to transmission, 

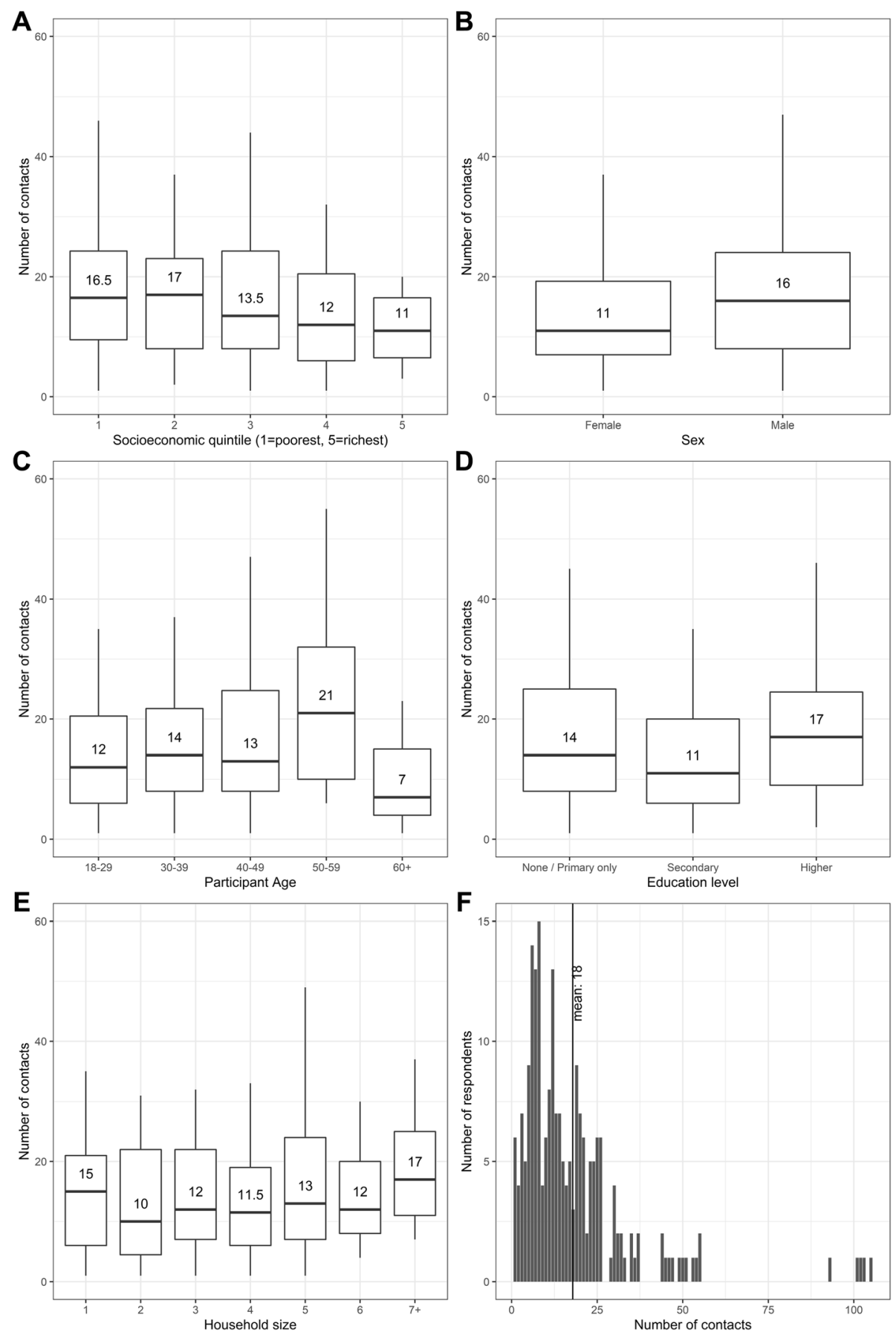

Fig. 1 Median number of direct contacts (physical and non-physical) by a socioeconomic status quintile, $\mathbf{b}$ gender, $\mathbf{c}$ respondent age, d education level, and e household size. Each panel shows the median, hinges (25th and 75th percentiles), and whiskers representing upper and lower adjacents. Outliers are not displayed in boxplots for scale; these are plotted in $\mathbf{f}$ showing the distribution of the number of direct contacts reported

or either 3.9 or 4.8 assuming all contacts are equally risky. As shown in Additional file 4, assuming that children are half as susceptible to SARS-CoV-2 infection compared to adults has little impact on $R_{0}$ estimates.

\section{Discussion}

COVID-19 control measures in informal settlements appear to have led to a large reduction in social contacts. We find a $62-67 \%$ reduction in eigenvalues of contact matrices depending on the pre-COVID-19 matrix used; 


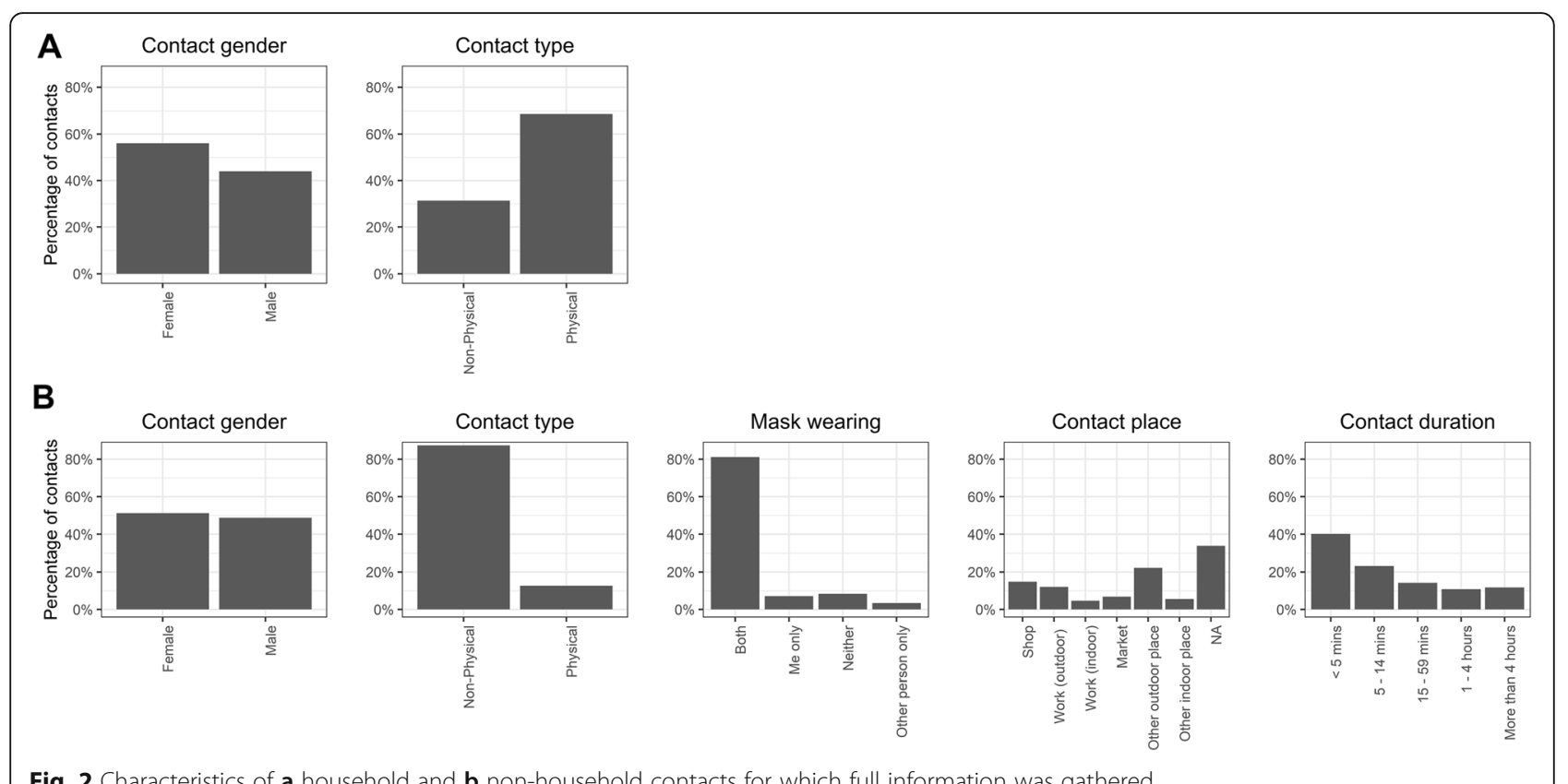

Fig. 2 Characteristics of $\mathbf{a}$ household and $\mathbf{b}$ non-household contacts for which full information was gathered

assuming an $R_{0}$ of 2.6, this would translate to an $R_{0}$ of between 0.5 and 0.7 at the time of data collection. By contrast, simulation estimates of the $R_{0}$ in an unmitigated COVID-19 epidemic in Kenya were between 1.78 (95\% CI 1.44-2.14) and 3.46 (95\% CI 2.81-4.17) [27]. The $R_{0}$ we estimate here is consistent with the slow growth of the Kenyan epidemic to-date compared to epidemics in China and Europe. The large reductions in contacts we estimate are of similar magnitude to those seen in both the UK [13] (74\% reduction in contacts), Wuhan and Shanghai [11] (86\% reduction), and the USA (70\% reduction) [12]. We are not aware of any comparable post-lockdown studies from low- or middle-income settings to-date, including sub-Saharan Africa.

Considerable food and economic vulnerability was reported due to COVID-19 control measures. Over $80 \%$ of respondents reported a partial or complete loss of income, and three quarters reported eating less or skipping

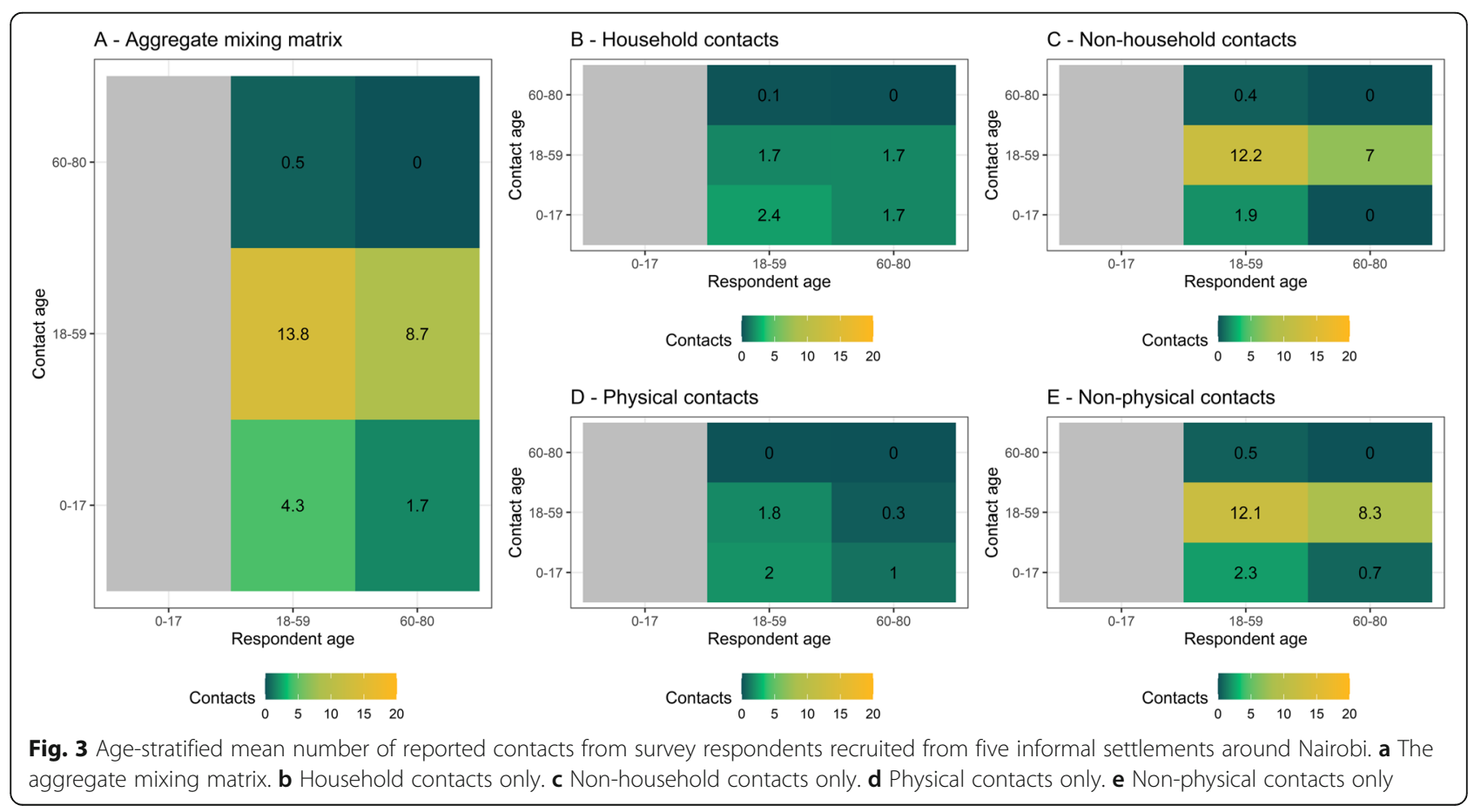




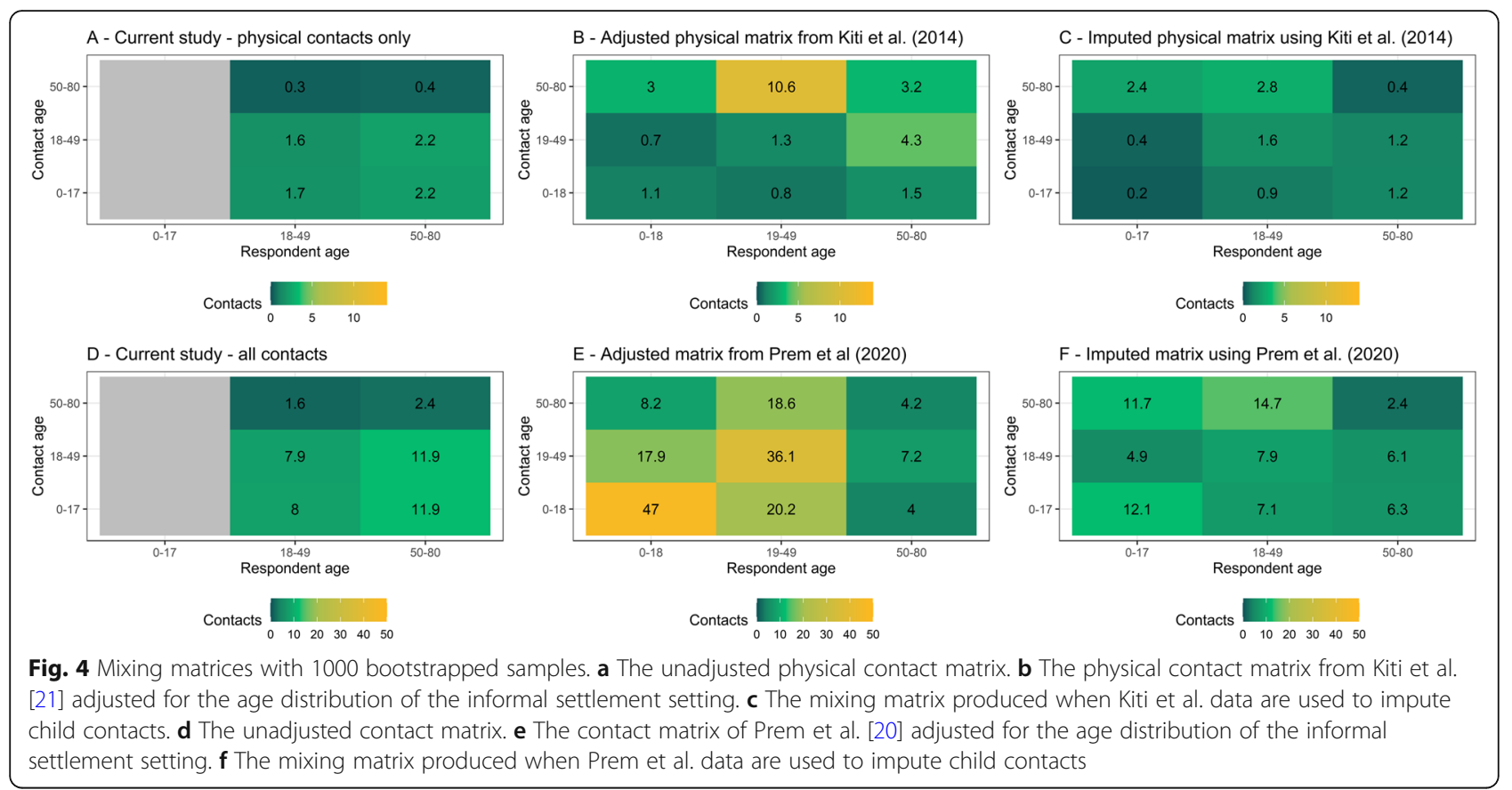

meals due to COVID-19. Households reported they were receiving some assistance, but that their biggest remaining unmet need was food. Although the prevalence of COVID-19 was low, and these factors can largely be attributed to control measures rather than illness from COVID-19 itself, it is important to recognise the counterfactual of no control measures is an unmitigated epidemic, and not an absence of these harms. The socioeconomic situation of informal settlements means that respondents may face greater economic precarity

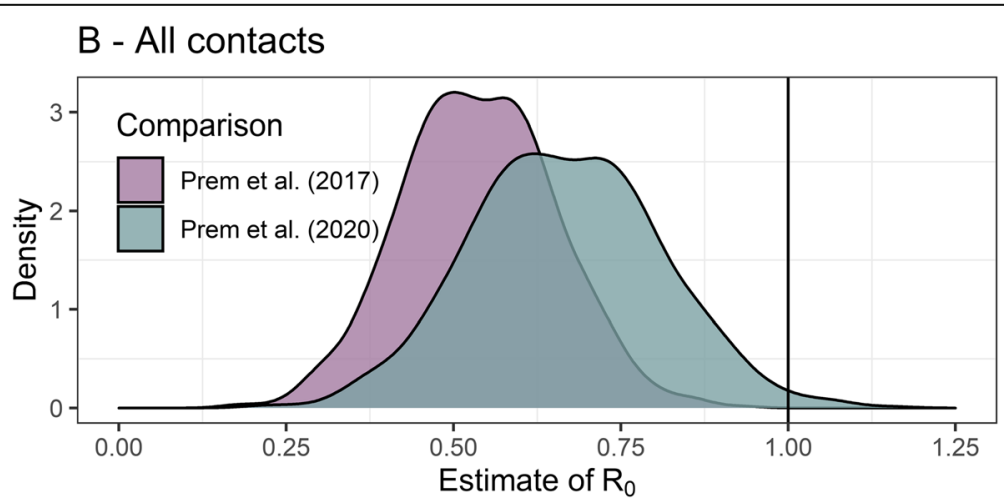

A - Physical contacts

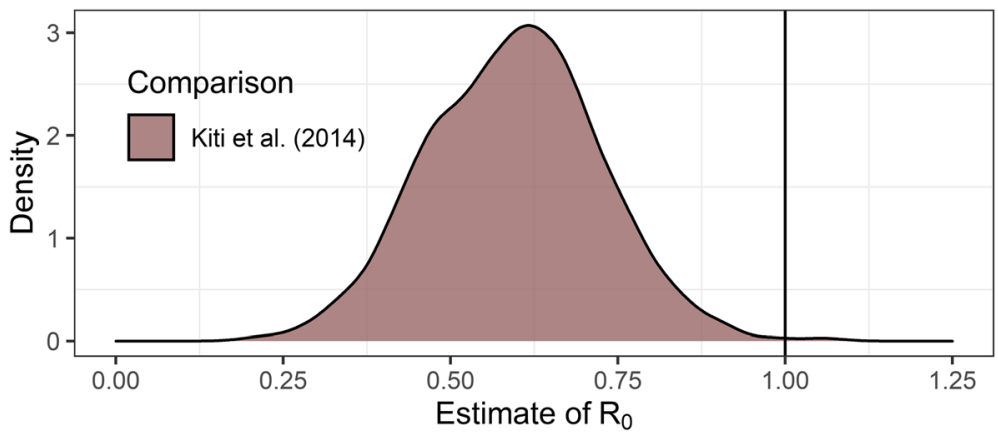

Fig. 5 Estimated value of $R_{0}$ at time of survey. $R_{0}$ assumed $\sim \operatorname{Norm}(2.6, S D=0.54)$ prior to control measures 
than residents of formal urban areas. Even within this sample, the poorest quintile of respondents reported 1.5 times as many contacts as the richest, suggesting an inequitable impact of COVID-19 transmission. This inequity would be exacerbated if socially patterned financial and access barriers inhibit the poor from seeking care for COVID-19 [28, 29]. Stringent control measures which cause economic and food insecurity are not likely to be sustainable in the long term if not accompanied by social protection mechanisms.

These estimates of $R_{0}$ are lower than those suggested by the linear growth of the epidemic in Kenya under control measures [1] which implies an $R_{0}$ of around 1, suggesting that there are other factors which influence transmission which we do not consider here. Contact patterns measured here only reflect community transmission, and if proportionately more infections occur due to contacts in non-community or clinical settings, then these estimates will overestimate the impact of control measures. As seen in many other settings, the number of reported cases is likely to be a significant underestimate of true cases given constraints in case finding and laboratory testing capacity: estimates suggest that during the study period, Kenya was detecting around $30 \%$ of symptomatic cases, compared to around $15 \%$ in the UK and the USA [30]. At present, evidence on how SARS-CoV-2 is transmitted is inconclusive [31, 32]; however, if fomites are a substantive cause of transmission in Kenyan informal settlements, then the current analysis will likely overstate the impact of control measures on $R_{0}$. We conject fomite transmission may be more likely in this setting due to high population density, and low and unequal access to water, sanitation, and hygiene amenities. Furthermore, the next-generation matrix approach of calculating $R_{0}$ which we use assumes uniform susceptibility and infectivity by age. In reality, younger people are less likely to acquire and transmit SARS-CoV-2 [26]. Because our contact data are collected in wide age ranges, if younger people have reduced contacts proportionately more than older people, our results may overestimate the impact on $R_{0}$. We found that assuming reduced susceptibility among children did not substantively change results.

Since data were collected for this study, the case numbers have continued to increase in Kenya. As of late July 2020, a progressive re-opening was occurring, including the lifting of movement restrictions in areas considered hotspots including parts of Nairobi and coastal counties, moving the start of nightly curfew from $7 \mathrm{pm}$ to $9 \mathrm{pm}$, and allowing the opening of places of worship, restaurants, and other places of communal gathering. Local air travel resumed on 15 July. Restrictions on the number of people allowed in such places remain, for example gatherings in places of worship are limited to 100 people for $1 \mathrm{~h}$, only for those over 13 or under 58 years of age. Schools remain closed until January 2021.

This study has a number of limitations. In the absence of baseline contact data (i.e. before control measures were put in place), we use empirical matrices from a different area of Kenya and synthetic matrices based on adjusting contact surveys from higher income countries to household and other characteristics in Kenya. Although we adjust these datasets by the age structure of the Kenyan population, other factors such as household size were not reported and may influence number of contacts and therefore pathogen transmission. The preCOVID-19 setting of Kiti et al. is very different to this sample, not least as estimates place population density around 24 times greater in informal settlements (Kibera, 55,000 persons $/ \mathrm{km}^{2}$ ) compared to urban Kilifi (2325 persons $/ \mathrm{km}^{2}$ ) [33]. Because we would expect contacts to be greater in more densely populated areas, the true reduction in contacts may be more than we estimate here. Although we have a range of background data on respondents from using existing sampling frames, households in the AGI-K and NITISU cohorts were initially selected as having an adolescent residing there in 2015 and 2018, respectively. Finally, although face mask use was reported by almost all participants, because of uncertainty in the effectiveness of masks in reducing SARS2-NCoV transmission, the impact of different types of face masks, and real-world adherence of mask users, $R_{0}$ calculations do not assume any protective effect from mask use.

Other social contact surveys have used a prospective study design, asking respondents to record contacts in a daily diary [34]. Because we asked respondents to recall contacts from the previous day, these data may be subject to recall bias, although it is not clear in which direction this may act. Furthermore, we impute adjusted child contacts using the comparison studies. An alternative approach, such as that taken by Kiti et al., would have been for respondents to record contacts for children in their household-arranging this was not possible during COVID-19 restrictions. To make the contact survey feasible for phone-based data collection, we simplified the tool for respondents who reported more than ten outside-household contacts. We are therefore limited to knowing these contacts' age and whether the contact was physical or non-physical. Contacts reported in this way were a substantial proportion (70\%) of the total sample. The main risk of bias from this may stem from respondents rounding up or down to anchor numbers (e.g. units of ten); Fig. 1e shows a few respondents cluster around 50 and 100 contacts. Overall, the loss of granularity was beneficial to reducing respondent burden. 
We do not calculate the net reproduction number, $R$, but because reported case numbers in Kenya are low, the proportion of the population that is no longer susceptible is likely minimal. We assume that direct contacts are a proxy for effective contacts and therefore transmission, and that transmissibility does not vary by age. In addition, we do not account for the very high proportion of respondents who report that they or their direct contacts wore face masks. Considering these factors would mean $R$ is below the $R_{0}$ estimated here.

\section{Conclusion}

Kenya has implemented strict control measures in response to the COVID-19 pandemic. This study highlights the difficult decisions policymakers face as we find that control measures are likely to have substantially reduced COVID-19 transmission, but also negatively impacted food and economic security of informal settlement residents. This is the first study to measure social contact patterns after COVID-19 control measures have been implemented in sub-Saharan Africa. There is evidence that impacts are inequitable, as the poorest quintile report 1.5 times more contacts than the richest quintile, and $86 \%$ of respondents reported complete or partial income losses. Negative and inequitable impacts on economic and food security may mean control measures are not sustainable in the longer term without social protection.

\section{Supplementary information}

Supplementary information accompanies this paper at https://doi.org/10. 1186/s12916-020-01779-4.

Additional file 1. Mixing data collection tool.
Additional file 2. Measurement of socioeconomic status, and food and economic security.

Additional file 3. Age adjustment.

Additional file 4. Age-susceptibility adjustment.

\section{Acknowledgements}

We are grateful for the excellent research assistance of Roseline Oguta, Hellen Collete Ochola, Emmanuel Mukabi, Carol Olela Adhiambo, Catherine Nduku Mwangi, Wesely Onsongo, Omachi Shawn Ambunya, James Joseph Okwogo, Esther Kariuki, Juliet Nduta Mwangi, Kadija Mohamed Ali, Vidah Achieng Oloo, and Lucy Kerubo Nyamwaro. We also acknowledge the contribution of Timothy Abuya, Faith Mbushi, Eva Muluve, James B. Tidwell, and Thoai D. Ngo. The following authors were part of the Centre for Mathematical Modelling of Infectious Disease COVID-19 working group. Each contributed in processing, cleaning, and interpretation of data; interpreted findings; contributed to the manuscript; and approved the work for publication: Nikos I Bosse, Graham Medley, Kathleen O'Reilly, Quentin J Leclerc, Mark Jit, Rachel Lowe, Nicholas G. Davies, Arminder K Deol, Gwenan M Knight, Megan Auzenbergs, W John Edmunds, Katherine E. Atkins, Thibaut Jombart, Rosalind M Eggo, Anna M Foss, Stefan Flasche, Carl A B Pearson, Emily S Nightingale, James D Munday, Sam Abbott, Billy J Quilty, Hamish P Gibbs, David Simons, Jon C Emery, Stéphane Hué, Yang Liu, Damien C Tully, Alicia Rosello, Simon R Procter, Akira Endo, Timothy W Russell, Eleanor M Rees, Charlie Diamond, Sophie R Meakin, Adam J Kucharski, Georgia R Gore-
Langton, Petra Klepac, Joel Hellewell, Fiona Yueqian Sun, Rein M G J Houben, C Julian Villabona-Arenas, Sebastian Funk, Samuel Clifford, and Oliver Brady.

\section{Authors' contributions}

Tool development and data collection: MQ, DM, BK, JP, and KA. Analysis: MQ, $K v Z, A G, K S, N M, K P$, and CIJ. First draft: MQ. Interpretation of findings, writing and critical revision of manuscript: $M Q, K v Z, A G, K S, N M, K P, E B, D M$, $B K, J P, C M M I D, W J E, C I J$, and KA. All authors have read and approved the manuscript.

\section{Funding}

The following funding sources are acknowledged as providing funding for the named authors. This research was partly funded by the Bill \& Melinda Gates Foundation (INV-003174: KP; INV-001754: MQ). DFID/Wellcome Trust (Epidemic Preparedness Coronavirus research programme 221303/Z/20/Z $\mathrm{KvZ}$ ). This research was partly funded by the National Institute for Health Research (NIHR) using UK aid from the UK Government to support global health research. The views expressed in this publication are those of the author(s) and not necessarily those of the NIHR or the UK Department of Health and Social Care (KvZ). ERC Starting Grant (757699: MQ). This project has received funding from the European Union's Horizon 2020 research and innovation programme - project EpiPose (101003688: KP, WJE). This research was partly funded by the Global Challenges Research Fund (GCRF) project "RECAP" managed through RCUK and ESRC (ES/P010873/1: AG, CIJ). This work was partly funded by United Kingdom Research and Innovation (UKRI) through the Medical Research Council (MRC) (MC_PC_19065: JE).

The following funding sources are acknowledged as providing funding for the working group authors. Alan Turing Institute (AE). BBSRC LIDP (BB/ M009513/1: DS). This research was partly funded by the Bill \& Melinda Gates Foundation (INV-003174: MJ, YL; NTD Modelling Consortium OPP1184344: CABP, GM; OPP1180644: SRP; OPP1183986: ESN; OPP1191821: KO'R, MA; INV001754: MQ). DFID/Wellcome Trust (Epidemic Preparedness Coronavirus research programme 221303/Z/20/Z: CABP). ERC Starting Grant (\#757699: JCE, RMGJH). This project has received funding from the European Union's Horizon 2020 research and innovation programme - project EpiPose (101003688: $\mathrm{MJ}, \mathrm{PK}, \mathrm{YL}$ ). This research was partly funded by the Global Challenges Research Fund (GCRF) project "RECAP" managed through RCUK and ESRC (ES/ P010873/1: TJ). HDR UK (MR/S003975/1: RME). Nakajima Foundation (AE). This research was partly funded by the National Institute for Health Research (NIHR) using UK aid from the UK Government to support global health research. The views expressed in this publication are those of the author(s) and not necessarily those of the NIHR or the UK Department of Health and Social Care (16/137/109: BJQ, CD, FYS, MJ, YL; Health Protection Research Unit for Modelling Methodology HPRU-2012-10096: NGD, TJ; PR-OD-1017-20002: AR). Royal Society (Dorothy Hodgkin Fellowship: RL; RP\EA\180004: PK). UK DHSC/UK Aid/NIHR (ITCRZ 03010: HPG). UK MRC (LID DTP MR/N013638/1: EMR, GRGL, QJL; MC_PC 19065: RME; MR/P014658/1: GMK). Authors of this research receive funding from UK Public Health Rapid Support Team funded by the UK Department of Health and Social Care ( $T \mathrm{~J})$. Wellcome Trust (206250/Z/17/Z: AJK, TWR; 206471/Z/17/Z: OJB; 208812/Z/17/Z: SC, SFlasche; 210758/Z/18/Z: JDM, JH, NIB, SA, SFunk, SRM). No funding (AKD, AMF, DCT, $\mathrm{SH})$.

Availability of data and materials

Data and code are fully available at https://github.com/mquaife/kenya_ mixing.

\section{Ethics approval and consent to participate}

The study was approved by the internal review board of the Population Council (study number 936), the ethics committee of the London School of Hygiene and Tropical Medicine (reference number 22294), and the AMREF Health Africa Ethics and Scientific Review Committee in Kenya (P803/2020). Verbal informed consent was obtained from all participants because written consent was not possible for phone-based interviews. The verbal consent process was approved by the three ethics committees named above.

Consent for publication

Not applicable

Competing interests

All authors declare no conflict of interest. 


\section{Author details}

${ }^{1}$ Faculty of Epidemiology and Population Health, London School of Hygiene and Tropical Medicine, London, UK. ${ }^{2}$ Faculty of Public Health and Policy, London School of Hygiene and Tropical Medicine, London, UK. ${ }^{3}$ Health Economics Research Unit, KEMRI-Wellcome Trust Research Programme, Nairobi, Kenya. ${ }^{4}$ Centre for Tropical Medicine, Nuffield Department of Clinical Medicine, University of Oxford, Oxford, UK. ${ }^{5}$ Population Council, Nairobi, Kenya. ${ }^{6}$ Population Council, New York, USA.

Received: 9 June 2020 Accepted: 9 September 2020

Published online: 05 October 2020

\section{References}

1. Dong E, Du H, Gardner L. An interactive web-based dashboard to track COVID-19 in real time. The Lancet infectious diseases. 2020;20(5):533-4.

2. Hale, T., et al. Oxford COVID-19 Government Response Tracker, Blavatnik School of Government. 2020 24/5/2020]; Available from: https:/www.bsg. ox.ac.uk/research/research-projects/coronavirus-government-responsetracker. Accessed 4 Aug 2020.

3. Cabore JW, Karamagi HC, Kipruto H, Asamani JA, Droti B, Seydi ABW, TitiOfei R, Impouma B, Yao M, Yoti Z, Zawaira F. The potential effects of widespread community transmission of SARS-CoV-2 infection in the World Health Organization African Region: a predictive model. BMJ Global Health. 2020;5(5):e002647.

4. Pearson CA, et al. Projected early spread of COVID-19 in Africa through 1 June 2020. Eurosurveillance. 2020;25(18):2000543.

5. Diop BZ, et al. The relatively young and rural population may limit the spread and severity of Covid-19 in Africa: a modelling study. BMJ Glob Health. 2020;5(5):e002699.

6. Gilbert $\mathrm{M}$, et al. Preparedness and vulnerability of African countries against importations of COVID-19: a modelling study. Lancet. 2020; 395(10227):871-7.

7. Nkengasong JN, Mankoula W. Looming threat of COVID-19 infection in Africa: act collectively, and fast. Lancet. 2020;395(10227):841-2.

8. Hopman J, Allegranzi B, Mehtar S. Managing COVID-19 in low-and middleincome countries. JAMA. 2020;323(16):1549-50.

9. Martinez-Alvarez M, et al. COVID-19 pandemic in west Africa. Lancet Glob Health. 2020;8(5):e631-2.

10. O'Reilly KM, Auzenbergs M, Jafari Y, Liu Y, Flasche S, Lowe R. Effective transmission across the globe: the role of climate in COVID-19 mitigation strategies. The Lancet Planetary Health. 2020;4(5):e172.

11. Zhang J, et al. Changes in contact patterns shape the dynamics of the COVID-19 outbreak in China: Science; 2020.

12. Feehan, D. and A. Mahmud, Quantifying interpersonal contact in the United States during the spread of COVID-19: first results from the Berkeley Interpersonal Contact Study. medRxiv, 2020. https:/www.medrxiv.org/ content/10.1101/2020.04.13.20064014v2.

13. Jarvis $\mathrm{Cl}$, et al. Quantifying the impact of physical distance measures on the transmission of COVID-19 in the UK. BMC Med. 2020;18:1-10.

14. Hoang $T$, et al. A systematic review of social contact surveys to inform transmission models of close-contact infections. Epidemiology. 2019;30(5): 723-36.

15. Dodd PJ, et al. Age-and sex-specific social contact patterns and incidence of Mycobacterium tuberculosis infection. Am J Epidemiol. 2016;183(2):156-66.

16. Johnstone-Robertson SP, et al. Social mixing patterns within a South African township community: implications for respiratory disease transmission and control. Am J Epidemiol. 2011;174(11):1246-55.

17. Melegaro A, Del Fava E, Poletti P, Merler S, Nyamukapa C, Williams J, Gregson S, Manfredi P. Social contact structures and time use patterns in the Manicaland Province of Zimbabwe. PloS one. 2017:12(1):e0170459.

18. Glynn JR, McLean E, Malava J, Dube A, Katundu C, Crampin AC, Geis S. Effect of Acute Illness on Contact Patterns, Malawi, 2017. Emerging infectious diseases. 2020;26(1):44.

19. Prem K, Cook AR, Jit M. Projecting social contact matrices in 152 countries using contact surveys and demographic data. PLoS Comput Biol. 2017;13(9): e1005697.

20. Prem, K., A.R. Cook, and M. Jit, Updated social contact matrices in 152 countries using contact surveys and demographic data. cmmid working paper, 2020
21. Kiti MC, Kinyanjui TM, Koech DC, Munywoki PK, Medley GF, Nokes DJ. Quantifying age-related rates of social contact using diaries in a rural coastal population of Kenya. PloS one. 2014;9(8):e104786.

22. Kenya National Bureau of Statistics, 2019 Kenya Population and Housing Census Volume III: distribution of population by age and sex 2019.

23. Klepac, P., et al., Contacts in context: large-scale setting-specific social mixing matrices from the BBC Pandemic project. medRxiv, 2020. https:// www.medrxiv.org/content/10.1101/2020.02.16.20023754v2.

24. Abbott, S., et al., Temporal variation in transmission during the COVID-19 outbreak. CMMID Repository, 2020. https://epiforecasts.io/covid/. Accessed 4 Aug 2020.

25. Flaxman $\mathrm{S}$, et al. Estimating the effects of non-pharmaceutical interventions on COVID-19 in Europe. Nature. 2020;584(7820):257-61.

26. Davies NG, Klepac P, Liu Y, et al. Age-dependent effects in the transmission and control of COVID-19 epidemics. Nat Med. 2020;26:1205-11. https://doi. org/10.1038/541591-020-0962-9.

27. Brand, S.P., et al., Forecasting the scale of the COVID-19 epidemic in Kenya. medRxiv, 2020. https://www.medrxiv.org/content/10.1101/2020.04.09.2005 $9865 \mathrm{v} 2$.

28. Kazungu JS, Barasa EW. Examining levels, distribution and correlates of health insurance coverage in Kenya. Tropical Med Int Health. 2017;22(9): $1175-85$.

29. llinca S, et al. Socio-economic inequality and inequity in use of health care services in Kenya: evidence from the fourth Kenya household health expenditure and utilization survey. Int J Equity Health. 2019;18(1):196.

30. Russell TW, et al. Using a delay-adjusted case fatality ratio to estimate under-reporting. London: Centre for Mathematical Modeling of Infectious Diseases Repository; 2020.

31. Kampf $\mathrm{G}$, et al. Persistence of coronaviruses on inanimate surfaces and their inactivation with biocidal agents. J Hosp Infect. 2020;104(3):246-51.

32. Goldman E. Exaggerated risk of transmission of COVID-19 by fomites. The Lancet Infectious Diseases. 2020;20(8):892-3.

33. Kenya National Bureau of Statistics, 2019 Kenya Population and Housing Census Volume II: distribution of population by administrative units 2019.

34. Mossong J, Hens N, Jit M, Beutels P, Auranen K, Mikolajczyk R, Massari M, Salmaso S, Tomba GS, Wallinga J, Heijne J. Social contacts and mixing patterns relevant to the spread of infectious diseases. PLoS Med. 2008;5(3): e74.

\section{Publisher's Note}

Springer Nature remains neutral with regard to jurisdictional claims in published maps and institutional affiliations.

\section{Ready to submit your research? Choose BMC and benefit from:}

- fast, convenient online submission

- thorough peer review by experienced researchers in your field

- rapid publication on acceptance

- support for research data, including large and complex data types

- gold Open Access which fosters wider collaboration and increased citations

- maximum visibility for your research: over $100 \mathrm{M}$ website views per year

At $\mathrm{BMC}$, research is always in progress.

Learn more biomedcentral.com/submissions 